\title{
AN INVERSE MAPPING THEOREM FOR ARC-ANALYTIC HOMEOMORPHISMS
}

\author{
TOSHIZUMI FUKUI \\ Department of Mathematics, Faculty of Science, Saitama University \\ 255 Shimo-Okubo, Urawa 338-8570, Japan \\ E-mail: tfukui@rimath.saitama-u.ac.jp \\ KRZYSZTOF KURDYKA \\ Laboratoire de Mathématiques, Université de Savoie \\ Campus Scientifique, 73376 Le Bourget-du-Lac Cedex, France \\ E-mail: kurdyka@univ-savoie.fr \\ LAURENTIU PAUNESCU \\ School of Mathematics and Statistics \\ University of Sydney, NSW 2006, Australia \\ E-mail: laurent@maths.usyd.edu.au
}

\begin{abstract}
We show that a subanalytic map-germ $\left(\mathbf{R}^{n}, 0\right) \rightarrow\left(\mathbf{R}^{n}, 0\right)$ which is arc-analytic and bi-Lipschitz has an arc-analytic inverse.

Let $U$ be an open subset of $\mathbf{R}^{n}$ or more generally a smooth real analytic variety. Following [8] we say that a map $f: U \rightarrow \mathbf{R}^{k}$ is arc-analytic if $f \circ \alpha$ is analytic for any analytic arc $\alpha:(-\varepsilon, \varepsilon) \rightarrow U$. In general these functions are very far from being analytic, in particular there are arc-analytic functions which are not subanalytic [9], not continuous [2], with a non-discrete singular set [10]. Hence it is natural to consider only arc-analytic maps with subanalytic graphs. In the late seventies T.-C. Kuo introduced the notion of blow-analytic mappings, i.e., mappings which become analytic after a composition with appropriate proper bi-meromorphic maps (e.g. composition of blowings-up with smooth centers). He suggested that the equivalence relation for germs of analytic functions, defined by blow-analytic homeomorphisms, should give "the canonical" strat-
\end{abstract}

2000 Mathematics Subject Classification: Primary 32B20; Secondary 14Pxx.

Key words and phrases: arc-analytic, Lipschitz, sub-analytic, homeomorphism.

The authors thank JSPS for making possible their collaboration.

The paper is in final form and no version of it will be published elsewhere. 
ification of the singular set of an analytic function. Actually he proved it for analytic families with isolated singularities [7].

Clearly any blow-analytic mapping is arc-analytic and subanalytic. The second author suggested that also the converse holds (published in [1], see also [11]). To be more precise one should assume that the function is arc-analytic and its graph is semi-algebraic with respect to the target variables.

Blow-analytic maps have been studied by several authors (see the survey [5]). One disappointing fact is that blow-analytic homeomorphisms are not necessarily bi-Lipschitz (locally) and therefore may not preserve the multiplicity of analytic curves [5], [13]. Clearly bi-Lipschitz arc-analytic homeomorphisms preserve the multiplicity of analytic curves (for convenience we prove it in Section 3). So now it becomes quite clear that something close to the bi-Lipschitz assumption must be added to the definition of the equivalence relation which is supposed to give the "the canonical" stratification of the singular sets of an analytic functions.

In this note we prove Implicit and Inverse Mapping Theorems in the bi-Lipschitz arc-analytic category. A version of an Inverse Mapping Theorem is given in [6] for some special blow-analytic analytic mappings (without assuming the bi-Lipschitz condition), see also [12] for similar results.

In Section 3 we discuss the problem of preserving multiplicities of curves by arcanalytic homeomorphisms. We also give an example which shows that it is hard to avoid the bi-Lipschitz assumption in the Inverse Mapping Theorem. Moreover we propose a conjecture, namely that the bi-Lipschitz arc-analytic homeomorphisms preserve the multiplicities of the real analytic sets. Finally, in Section 4, we give an effective characterization of the bi-Lipschitz map-germs.

1. Local version. We say that a map-germ $f:\left(\mathbf{R}^{n}, 0\right) \rightarrow\left(\mathbf{R}^{n}, 0\right)$ is bi-Lipschitz if there exist positive constants $c_{1}, c_{2}$ such that

$$
c_{1}\left|y-y^{\prime}\right| \leq\left|f(y)-f\left(y^{\prime}\right)\right| \leq c_{2}\left|y-y^{\prime}\right|, \quad \forall y, y^{\prime} \in\left(\mathbf{R}^{n}, 0\right) .
$$

We recall now a notion which is a set-theoretic counter part of arc-analyticity. Let $M$ be an analytic manifold, we say that $E \subset M$ is arcwise symmetric in $M$ (cf. [8]), if one of the following two equivalent conditions holds:

i) Int $\gamma^{-1}(E) \neq \emptyset \Longrightarrow \gamma(-1,1) \subset E$, for every analytic arc $\gamma:(-1,1) \rightarrow V \subset M$;

ii) $\gamma(-1,0) \subset E \Longrightarrow \gamma(-1,1) \subset E$, for every analytic arc $\gamma:(-1,1) \rightarrow V \subset M$.

Recall also that any arc-analytic mapping has arcwise symmetric graph (cf. [8]), but of course the converse is not true. In fact, take for example $f(x)=x^{5 / 3}, x \in \mathbf{R}$, which is a $C^{1}$ function with algebraic graph $y^{3}=x^{5}$ and obviously $f$ is not arc-analytic.

We state now the local version of our Implicit Function Theorem.

TheOREM 1.1. Let $F:\left(\mathbf{R}^{m} \times \mathbf{R}^{n}, 0\right) \rightarrow\left(\mathbf{R}^{n}, 0\right)$ be a subanalytic map-germ which is arc-analytic. Assume that there exist positive constants $c_{1}, c_{2}$ such that

$$
c_{1}\left|y-y^{\prime}\right| \leq\left|F(x, y)-F\left(x, y^{\prime}\right)\right| \leq c_{2}\left|y-y^{\prime}\right|, \quad \forall x \in\left(\mathbf{R}^{m}, 0\right), \forall y, y^{\prime} \in\left(\mathbf{R}^{n}, 0\right) .
$$


Then there exists an arc-analytic and subanalytic map $\tau:\left(\mathbf{R}^{m}, 0\right) \rightarrow\left(\mathbf{R}^{n}, 0\right)$ such that

$$
\{F(x, y)=0\}=\{y=\tau(x)\}
$$

near the origin of $\mathbf{R}^{m} \times \mathbf{R}^{n}$.

Put $f_{x}(y)=F(x, y)$, by (1.1) we know that each $f_{x}$ is continuous and injective in a small neighborhood of the origin. This fact and our assumption that $F(0,0)=0$ will imply the existence of a unique continuous solution $\tau(x)$ of $f_{x}(y)=0$. This is a purely topological property (it involves only Brouwer's theorem on the invariance of domain). For completeness, we give a proof of this fact here.

LEMma 1.2. Let $F:\left(\mathbf{R}^{m} \times \mathbf{R}^{n}, 0\right) \rightarrow\left(\mathbf{R}^{n}, 0\right)$ be a continuous map. Assume that there exists a positive constant $c$ such that

$$
c\left|y-y^{\prime}\right| \leq\left|F(x, y)-F\left(x, y^{\prime}\right)\right|, \quad \forall x \in\left(\mathbf{R}^{m}, 0\right), \forall y, y^{\prime} \in\left(\mathbf{R}^{n}, 0\right) .
$$

Then there exists a unique continuous map $\tau:\left(\mathbf{R}^{m}, 0\right) \rightarrow\left(\mathbf{R}^{n}, 0\right)$ satisfying $(1.2)$.

Proof. We choose $D$ an open neighborhood of the origin in $\mathbf{R}^{m+n}$. We denote by $D_{x}$ the intersection $D \cap\left(\{x\} \times \mathbf{R}^{n}\right)$ for $x$ in a small neighborhood of the origin. We define

$$
\varepsilon(x)=\operatorname{dist}\left(f_{x}(0), \partial f_{x}\left(D_{x}\right)\right),
$$

where $\partial f_{x}\left(D_{x}\right)$ means the boundary of $f_{x}\left(D_{x}\right)$.

By the continuity of $F$ and condition (1.3) it is easily seen that there is $\lambda>0$ such that $\varepsilon(x) \geq \lambda$ in a neighborhood of $0 \in \mathbf{R}^{m}$. Choose $\delta>0$ such that $\left|f_{x}(0)\right| \leq \frac{\lambda}{2}$ for all $x$ with $|x|<\delta$. Thus we obtain that $0 \in f_{x}\left(D_{x}\right)$. Since $f_{x}$ is injective there is a unique point $\tau(x) \in \mathbf{R}^{n}$ such that $f_{x}(\tau(x))=F(x, \tau(x))=0$. Note that the graph of $\tau$ is compact so $\tau$ is continuous.

Proof of Theorem 1.1. The map $\tau$ is subanalytic, because its graph coincides with the zero set of $F$. We have to show that $\tau$ is arc-analytic.

Let $\gamma:(\mathbf{R}, 0) \rightarrow\left(\mathbf{R}^{m}, 0\right)$ be a germ of an analytic arc. By the definition of arcanalyticity we have to prove that $\tau \circ \gamma$ is analytic. Replacing $F(x, y)$ by $F(\gamma(t), y)$ we see that it is enough to show the following.

Proposition 1.3. Let $F:\left(\mathbf{R}^{m} \times \mathbf{R}^{n}, 0\right) \rightarrow\left(\mathbf{R}^{n}, 0\right)$ be as in Theorem 1.1 and assume $m=1$. Then the corresponding $\tau$ is analytic near the origin.

Proof. Because $F$ is arc-analytic and subanalytic, its graph is arcwise symmetric. So it follows that $\{F(x, y)=0\}$ is arcwise symmetric and subanalytic (see [8]). Since $\{F(x, y)=0\}$ is the graph of a subanalytic function in one variable its dimension is 1 . Recall that any arcwise symmetric subanalytic set of dimension 1 is actually analytic ([8]). Hence $\{F(x, y)=0\}$ is analytic in a neighborhood of $(0,0) \in \mathbf{R} \times \mathbf{R}^{n}$. Moreover its germ at the origin is irreducible since it is a germ of the graph of function $\tau$. Let us write $\tau=\left(\tau_{1}, \ldots, \tau_{n}\right)$. Each component has a Newton-Puiseux expansion

$$
\tau_{i}(x)=\sum_{\nu \in \mathbf{N}} a_{\nu}^{i} x^{\nu / q}, \quad i=1, \ldots, n,
$$

for some positive integer $q$ and $x>0$. 
Because the germ of $\{F(x, y)=0\}$ is analytic and irreducible if we show that $\tau(x)$, $x>0$, can be extended to an analytic map in a neighborhood of $0 \in \mathbf{R}$, this extension must coincide with $\tau$ itself. Hence we have to prove that there are no terms with noninteger exponents in the expansion (1.4).

Let $p_{i}(x), i=1, \ldots, n$, be the analytic part of the expansion (1.4). Performing the analytic change of variable $\left(x, y_{1}, \ldots, y_{n}\right) \mapsto\left(x, y_{1}+p_{1}(x), \ldots, y_{n}+p_{n}(x)\right)$ we may assume that all the expansions in (1.4) start with fractional exponents if not identical zero. If all of them are zero we are done. Suppose that they are not identically zero. By assumption, there exist positive numbers $c_{1}, c_{2}$ (independent of $x$ ) such that

$$
c_{1}|y| \leq|F(x, y)-F(x, 0)| \leq c_{2}|y|, \quad \forall y \in\left(\mathbf{R}^{n}, 0\right), \forall x \in(\mathbf{R}, 0) .
$$

If we replace $y$ by $\tau(x)$ we obtain

$$
c_{1}|\tau(x)| \leq|F(x, \tau(x))-F(x, 0)|=|F(x, 0)| \leq c_{2}|\tau(x)| .
$$

By assumption, $F(x, 0)$ is analytic in $x$ and vanishes at $x=0$. These two inequalities show that $\tau$ cannot start with a non-integer power and this finishes the proof.

TheOrem 1.4. Let $f:\left(\mathbf{R}^{n}, 0\right) \rightarrow\left(\mathbf{R}^{n}, 0\right)$ be a subanalytic map which is arc-analytic. If $f$ is bi-Lipschitz, then $f$ has an inverse which is arc-analytic (and subanalytic).

Proof. We apply Theorem 1.1 to the map $F(x, y)=x-f(y)$.

Since, by [1], every semi-algebraic and arc-analytic mapping is actually blow-analytic, we obtain

Theorem 1.5. Let $f:\left(\mathbf{R}^{n}, 0\right) \rightarrow\left(\mathbf{R}^{n}, 0\right)$ be a semi-algebraic map which is blowanalytic. If $f$ is bi-Lipschitz, then $f$ has an inverse which is blow-analytic.

2. Global version. The same proofs show the following global versions of Theorems $1.1,1.4,1.5$.

TheORem 2.1. Let $F: \mathbf{R}^{m} \times \mathbf{R}^{n} \rightarrow \mathbf{R}^{n}$ be a subanalytic map which is arc-analytic. If there are positive numbers $c_{1}, c_{2}$ such that

$$
c_{1}\left|y-y^{\prime}\right| \leq\left|F(x, y)-F\left(x, y^{\prime}\right)\right| \leq c_{2}\left|y-y^{\prime}\right|, \quad \forall x \in \mathbf{R}^{m}, \forall y, y^{\prime} \in \mathbf{R}^{n},
$$

then there exists an arc-analytic and subanalytic map $\tau: \mathbf{R}^{m} \rightarrow \mathbf{R}^{n}$ such that

$$
\{F(x, y)=0\}=\{y=\tau(x)\} \quad \text { in } \mathbf{R}^{m} \times \mathbf{R}^{n} .
$$

TheOREM 2.2. Let $f: \mathbf{R}^{n} \rightarrow \mathbf{R}^{n}$ be a subanalytic map which is arc-analytic. If $f$ is bi-Lipschitz, then $f$ has an inverse which is arc-analytic (and subanalytic).

Similarly in the semi-algebraic case we have

THEOREM 2.3. Let $f: \mathbf{R}^{n} \rightarrow \mathbf{R}^{n}$ be a semi-algebraic map which is blow-analytic. If $f$ is bi-Lipschitz, then $f$ has an inverse which is blow-analytic (and semi-algebraic). 
3. Preserving orders and multiplicities. The role of the bi-Lipschitz condition in the above theorems is crucial, as is shown by the example given at the end of the section. Actually one could try to weaken the bi-Lipschitz condition (1.1) in the Inverse Mapping Theorem 1.4 by fixing one variable. More precisely we say that a map-germ $f:\left(\mathbf{R}^{n}, y\right) \rightarrow\left(\mathbf{R}^{k}, f(y)\right)$ is semi-bi-Lipschitz at the point $y$ if there exist positive constants $c_{1}, c_{2}$, such that

$$
c_{1}\left|y-y^{\prime}\right| \leq\left|f(y)-f\left(y^{\prime}\right)\right| \leq c_{2}\left|y-y^{\prime}\right|, \quad \forall y^{\prime} \in\left(\mathbf{R}^{n}, y\right) .
$$

What is interesting about semi-bi-Lipschitz and arc-analytic maps is that they preserve the multiplicities of the real analytic curves. As a piece of terminology we recall that if $\gamma:(\mathbf{R}, 0) \rightarrow\left(\mathbf{R}^{n}, 0\right)$ is an analytic (non-constant) arc then there exists a unique $m \in \mathbf{N}$ such that

$$
a t^{m} \leq|\gamma(t)| \leq b t^{m}
$$

for some $0<a \leq b$. We call the integer $m=\operatorname{ord}_{0} \gamma$ the $\operatorname{order}$ of $\gamma($ at 0$)$. Assume moreover that $\gamma$ is injective, then $\Gamma$, the image of $\gamma$, is a well defined irreducible germ of an analytic set of dimension 1 , and we shall say for short that $\Gamma$ is a germ of an analytic curve. So $\Gamma$ has a well defined multiplicity at $0 \in \mathbf{R}^{n}$, and we denote it by mult ${ }_{0} \Gamma$. Recall that

$$
\operatorname{mult}_{0} \Gamma=\inf \left\{\operatorname{ord}_{0} \gamma: \gamma \text { is a parametrization of } \Gamma\right\} \text {. }
$$

Moreover, mult $_{0} \Gamma=\operatorname{ord}_{0} \gamma$ iff the complexification of $\gamma$ is injective.

Clearly we have:

Proposition 3.1. Let $f:\left(\mathbf{R}^{n}, 0\right) \rightarrow\left(\mathbf{R}^{k}, 0\right)$ be the germ of a semi-bi-Lipschitz, arcanalytic mapping. Then $f$ preserves the orders of analytic arcs, i.e., $\operatorname{ord}_{0}(f \circ \gamma)=\operatorname{ord}_{0} \gamma$ for any analytic arc $\gamma:(\mathbf{R}, 0) \rightarrow\left(\mathbf{R}^{n}, 0\right)$.

As a consequence we obtain

Proposition 3.2. Let $f:\left(\mathbf{R}^{n}, 0\right) \rightarrow\left(\mathbf{R}^{n}, 0\right)$ be the germ of a subanalytic homeomorphism. Assume that $f$ and $f^{-1}$ are arc-analytic. Then $f$ is semi-bi-Lipschitz if and only if for any germ of irreducible analytic curve $\Gamma$, the multiplicities of $\Gamma$ and $f(\Gamma)$ are the same.

Proof. Let $\gamma(t)$ be an analytic parametrization of $\Gamma$ such that its complexification is injective. Suppose that $f$ is semi-bi-Lipschitz, so by Proposition 3.1 and (3.3)

$$
\operatorname{mult}_{0} \Gamma=\operatorname{ord}_{0} \gamma=\operatorname{ord}_{0}(f \circ \gamma) \geq \operatorname{mult}_{0} f(\Gamma) .
$$

The same argument applied to $f^{-1}$ gives the inverse inequality, so mult $\Gamma=$ mult $_{0} f(\Gamma)$.

To prove the converse let us assume that $f$ preserves the multiplicities of analytic curves but it is not semi-bi-Lipschitz at the origin. By the curve selection lemma there exists an analytic arc $\gamma(t), \gamma(0)=0$, such that

$$
\lim _{t \rightarrow 0} \frac{|f(\gamma(t))|}{|\gamma(t)|}=0 \text { or } \infty .
$$

We may suppose that the limit equals $\infty$, otherwise we replace $f$ by $f^{-1}$. We may also choose $\gamma$ such that mult ${ }_{0} \Gamma=\operatorname{ord}_{0} \gamma$. So, as above, we get

$$
\operatorname{mult}_{0} \Gamma=\operatorname{ord}_{0} \gamma>\operatorname{ord}_{0}(f \circ \gamma) \geq \operatorname{mult}_{0} f(\Gamma),
$$

which is a contradiction. 
Remark. Recently G. Comte, P. D. Milman, and D. Trotman [4] observed that under an extra condition, semi-bi-Lipschitz homeomorphisms preserve multiplicities of the complex hypersurfaces, which is a special case of the Zariski Conjecture about topological invariance of the multiplicities of the complex hypersurfaces.

Proposition 3.2 led us to the following conjecture.

CONJECTURE 3.3. Bi-Lipschitz arc-analytic homeomorphisms preserve the multiplicities of the real analytic sets. More precisely: let $f:\left(\mathbf{R}^{n}, 0\right) \rightarrow\left(\mathbf{R}^{n}, 0\right)$ be the germ of a subanalytic, arc-analytic, bi-Lipschitz homeomorphism, and let $X, Y$ be two irreducible analytic germs. Suppose that $Y=f(X)$, then mult $_{0} X=$ mult $_{0} Y$.

Note that it is not even known that the multiplicity is a bi-Lipschitz invariant of the complex hypersurfaces. On the other hand in the real case clearly multiplicity is not a bi-Lipschitz invariant. The curve $x^{7}=y^{3}$ is bi-Lipschitz equivalent to the line $y=0$ but not arc-analytic bi-Lipschitz equivalent.

As the following example shows, the Inverse Mapping Theorem 1.4 does not hold if we replace the bi-Lipschitz condition (1.1) by the semi-bi-Lipschitz condition (3.1). Hence in some sense our results are optimal.

EXAmple 3.4. Let $h: \mathbf{R}^{2} \rightarrow \mathbf{R}^{2}$ be the homeomorphism defined by

$$
h(x, y)=\left(\frac{x^{2}+9 y^{2}}{x^{2}+y^{2}} x, y\right) .
$$

Then $h$ is Lipschitz in a neighborhood of the origin, and it satisfies also the global inequality

$$
\left|(x, y)-\left(0, y^{\prime}\right)\right| \leq\left|h(x, y)-h\left(0, y^{\prime}\right)\right|
$$

but the inverse of $h$ is not arc-analytic.

Indeed, consider the curve $a(t)=(t \sqrt{3(9-t)}, t)$. We can see that $h^{-1}(a(t))$ is not analytic (it comes to show that the equation $X^{3}+X t^{3}+2 \frac{\sqrt{3(9-t)}}{9} t^{4}=0$ has no analytic solutions, and this can be done easily by inspecting the possible orders which may appear). From this last example we can see that the multiplicity of the analytic set $h^{-1}(a(t))$ is not preserved under $h$ (the image being a smooth curve).

4. Discussion of the bi-Lipschitz condition. In this section we shall give a simple and efficient characterization of subanalytic bi-Lipschitz map germs.

Let $U$ be an open and convex subset of $\mathbf{R}^{n}$ and let $f: U \rightarrow \mathbf{R}$ be a continuous and subanalytic function. Recall that $f$ is differentiable (even analytic) outside a closed nowhere dense subanalytic set $Z \subset U$. By the Mean Value Theorem and continuity of $f$ we obtain

LEMMA 4.1. The function $f$ is Lipschitz if and only if all the partial derivatives of $f$ are bounded in $U \backslash Z$, that is, there exists a constant $M>0$ such that $\left|\frac{\partial f}{\partial x_{i}}(x)\right| \leq M$ for all $x \in U \backslash Z$ and $i=1, \ldots, n$. 
We deduce now a characterization of the bi-Lipschitz map-germs:

Proposition 4.2. Let $f:\left(\mathbf{R}^{n}, 0\right) \rightarrow\left(\mathbf{R}^{n}, 0\right)$ be a continuous subanalytic map-germ, and let $Z$ denote the set of points where $f$ is not differentiable (recall that $Z$ is subanalytic and nowhere dense). Then $f$ is bi-Lipschitz if and only if it verifies the following three conditions:

(i) $f$ is injective,

(ii) the partial derivatives of $f$ are bounded,

(iii) there exists a constant $c>0$ such that $c \leq|\mathrm{Jac} f(x)|$, for any $x \notin Z$ close to the origin, where Jac $f(x)$ is the Jacobian of $f$ at $x$.

Proof. Assume that $f$ is bi-Lipschitz, then conditions (i), (ii) follow immediately. Let $A$ be the Jacobian matrix of $f$ at $x$. Since $f^{-1}$ is Lipschitz there exists a constant $b>0$, independent of $x$, such that $\left\|A^{-1}\right\| \leq b$. By elementary linear algebra, writing the module of the determinant as the volume of the image of the unit ball, we get

$$
|\operatorname{det} A| \geq\left\|A^{-1}\right\|^{-n} \geq b^{-n}
$$

(here it is convenient to take the "maximum" norm on $\mathbf{R}^{n}$ ). So condition (iii) follows with $c=b^{-n}$.

To prove the converse assume that $f$ verifies the above three conditions. It is enough to check that $f^{-1}$ is Lipschitz, that is, by Lemma $4.1\left(f^{-1}\right.$ is already continuous subanalytic) that the partial derivatives of $f^{-1}$ are bounded. But this easily follows from the formula for the inverse matrix, since $|\operatorname{Jac} f(x)|$ is bounded away from zero and also the partial derivatives of $f$ are bounded from above.

REMARK. If $f$ is as above and bi-Lipschitz it follows that there exist constants $c_{1}>0$, $c_{2}>0$ such that $c_{1} \leq|\operatorname{Jac} f(x)| \leq c_{2}$, for any $x \notin Z$ close to the origin, where $\operatorname{Jac} f(x)$ is the Jacobian of $f$ at $x$.

We also remark that our results above hold under the weaker assumption that $f$ is continuous and admits the Mean Value Theorem outside a thin set in the domain (in the subanalytic case this is automatically satisfied). As a comparison, it is worth to mention an interesting sufficient condition for a map-germ $f:\left(\mathbf{R}^{n}, 0\right) \rightarrow\left(\mathbf{R}^{n}, 0\right)$ to be bi-Lipschitz, given in [3] (Theorem 3.12). It claims that if the closure of the convex hull of the set $\{d f(x): x \in U \backslash Z\}$, where $U$ is a sufficiently small neighborhood of the origin, is compact and contains no singular matrix, then $f$ is bi-Lipschitz.

It is worth noticing that all assumptions in Proposition 4.2 are necessary, as the following example and remark show.

EXAMPLE 4.3. Let $n$ be a non-negative integer. Let $f:(\mathbf{C}, 0) \rightarrow(\mathbf{C}, 0)$ be the map defined by $z \mapsto z^{n+1} / \bar{z}^{n}$. We identify $\mathbf{C}$ with $\mathbf{R}^{2}$. The map $f$ is blow-analytic and hence arc-analytic and its graph is subanalytic. Moreover its Jacobian is constant:

$$
\operatorname{Jac}(f)=\left|\begin{array}{cc}
(n+1) z^{n} / \bar{z}^{n} & -n z^{n+1} / \bar{z}^{n+1} \\
-n \bar{z}^{n+1} / z^{n+1} & (n+1) \bar{z}^{n} / z^{n}
\end{array}\right|=(n+1)^{2}-n^{2}=2 n+1
$$

Lemma 4.1 shows that $f$ is Lipschitz. Since the mapping degree of $f$ is $2 n+1, f$ does not have an inverse and hence it cannot be bi-Lipschitz. 
Remark. Also our Example 3.4 shows that it is not enough to have only $f=h$ injective and Lipschitz (here again $f$ is blow-analytic).

If we consider again our Example 3.4 and take $f=h^{-1}$, we can see that it does not suffice that $f$ is injective and $1 / 9 \leq|\operatorname{Jac} f(x)|$ (note in this case $f$ is not arc-analytic).

However in the setting of arc-analytic maps, it is not clear for us if all the conditions in the Proposition 4.2 are necessary, in particular we have the following question.

QUESTION 4.4. Does there exist an arc-analytic and subanalytic map-germ $f:\left(\mathbf{R}^{n}, 0\right) \rightarrow\left(\mathbf{R}^{n}, 0\right)$ with the following properties:

(i) there exists a positive number $c$ such that

$$
c\left|y-y^{\prime}\right| \leq\left|f(y)-f\left(y^{\prime}\right)\right|, \quad \forall y, y^{\prime} \in\left(\mathbf{R}^{n}, 0\right),
$$

(ii) $f$ is not Lipschitz?

\section{References}

[1] E. Bierstone, P. D. Milman, Arc-analytic functions, Invent. Math. 101 (1990), 411-424.

[2] E. Bierstone, P. D. Milman, A. Parusiński, A function which is arc-analytic but not continuous, Proc. Amer. Math. Soc. 113 (1991), 419-423.

[3] F. H. Clarke, Yu. S. Ledyaev, R. J. Stern, P. R. Wolenski, Nonsmooth Analysis and Control Theory, Grad. Texts in Math. 178, Springer, New York, 1998.

[4] G. Comte, P. D. Milman, D. Trotman, On Zariski's multiplicities problem, Proc. Amer. Math. Soc. 130 (2002), 2045-2048.

[5] T. Fukui, S. Koike, T.-C. Kuo, Blow-analytic equisingularities, properties, problems and progress, in: Real Analytic and Algebraic Singularities, Pitman Res. Notes Math. Ser. 381, Longman, Harlow, 1998, 8-29.

[6] T. Fukui, T.-C. Kuo, L. Paunescu, Constructing blow-analytic isomorphisms, Ann. Inst. Fourier (Grenoble) 51 (2001), 1071-1087.

[7] T.-C. Kuo, On classification of real singularities, Invent. Math. 82 (1985), 257-262.

[8] K. Kurdyka, Ensembles semi-algébriques symétriques par arcs, Math. Ann. 282 (1988), 445-462.

[9] K. Kurdyka, A counterexample to subanalyticity of an arc-analytic function, Ann. Polon. Math. 55 (1991), 241-243.

[10] K. Kurdyka, An arc-analytic function with nondiscrete singular set, Ann. Polon. Math. 59 (1994), 251-254.

[11] A. Parusiński, Subanalytic functions, Trans. Amer. Math. Soc. 344 (1994), 583-595.

[12] L. Paunescu, Implicit Function Theorem for locally blow-analytic functions, Ann. Inst. Fourier (Grenoble) 51 (2001), 1089-1100.

[13] L. Paunescu, An example of blow-analytic homeomorphism, in: Real Analytic and Algebraic Singularities, Pitman Res. Notes Math. Ser. 381, Longman, Harlow, 1998, 62-63. 